\title{
Inhibition of XIAP increases carboplatin sensitivity in ovarian cancer
}

This article was published in the following Dove Press journal:

OncoTargets and Therapy

\author{
Yiping Zhang ${ }^{1,2}$ \\ Furong Huang ${ }^{3}$ \\ Qingyu Luo $^{3}$ \\ Xiaowei $\mathrm{Wu}^{3}$ \\ Zhihua $\mathrm{Liu}^{3}$ \\ Hongyan Chen ${ }^{3}$ \\ Yinghui Huang' \\ 'Cancer Institute, College of Life \\ Science and Bioengineering, Beijing \\ University of Technology, Beijing, \\ China; ${ }^{2}$ China National Center for \\ Biotechnology Development, Beijing, \\ China; ${ }^{3}$ The State Key Laboratory \\ of Molecular Oncology, National \\ Cancer Center/National Clinical \\ Research Center/Cancer Hospital, \\ Chinese Academy of Medical Sciences \\ and Peking Union Medical College, \\ Beijing, China
}

Purpose: Carboplatin is a first-line treatment for ovarian cancer. However, most patients develop resistance and undergo disease recurrence. This study aims to explore the relationship between the expression of X-linked inhibitor of apoptosis protein (XIAP) and carboplatin sensitivity in ovarian cancer.

Patients and methods: We examined the expression of XIAP in ovarian cancer by immunochemistry. Next, we investigated the role of XIAP in regulating carboplatin sensitivity in ovarian cancer ES2 and 3AO cells through Cell Counting Kit-8 cell viability assay and fluorescein isothiocyanate-Annexin V/propidium iodide apoptosis assay. Expression of apoptotic effectors was measured by Western blot.

Results: The immunochemistry results showed that high XIAP expression levels inversely correlated with carboplatin response $(P=0.03)$ and progression-free survival $(P=0.0068)$ in patients with ovarian cancer. Knockdown of XIAP repressed the cell viabilities in the carboplatin-treated cells and increased carboplatin-induced caspase activation. In summary, our data show that XIAP mediates carboplatin sensitivity of ovarian cancer.

Conclusion: In summary, our data show that XIAP mediates carboplatin sensitivity of ovarian cancer and XIAP may be a novel target for the treatment of carboplatin-resistant ovarian cancer. Keywords: XIAP, carboplatin, chemosensitivity, ovarian cancer

\section{Introduction}

Ovarian cancer is the most lethal type of malignant tumors of the female reproductive system, and the patient's 5-year survival rate is relatively low and has the highest mortality rate. Platinum compounds have been proved to be the most effective drugs for the treatment of ovarian carcinoma. Carboplatin has long been established as a first-line drug for the treatment of ovarian carcinoma, ${ }^{1}$ but the resistance of patients to platinum compounds is a major challenge in the treatment of ovarian cancer. ${ }^{2}$ So far, there is no very effective solution to this problem. ${ }^{3,4}$ Therefore, it is important to identify markers for predicting response to carboplatin, which may allow for the development of therapeutic strategies for overcoming chemoresistance.

Inhibitor of apoptosis protein (IAP) family has been reported to promote tumor formation and metastasis. ${ }^{5,6} \mathrm{X}$-linked inhibitor of apoptosis protein (XIAP) is a member of the IAP family. XIAP not only exerts an anti-apoptotic function but also inhibits autophagy via XIAP-Mdm2-p53 signaling. ${ }^{7}$ In addition, XIAP has been involved in regulating innate immune responses by mediating NOD signaling through interaction with RIP $2 .{ }^{8}$ Also, XIAP confers resistance to some chemotherapeutic drugs in various types of cancer including ovarian cancer. ${ }^{9-14}$ For example, phenoxodiol, Piceatannol, and HtrA1 enhance cisplatin sensitivity in ovarian cancer via the degradation of 
XIAP. ${ }^{9-11}$ However, there have been no studies that investigated the role of XIAP in conferring carboplatin sensitivity in ovarian cancer.

In the present study, we examined the expression of XIAP in clinical ovarian cancer samples (151 cases) and analyzed the relationships between XIAP expression and clinicopathological features of ovarian cancer. Furthermore, we investigated the functional role of XIAP on carboplatin sensitivity at the cellular and molecular level.

\section{Materials and methods}

\section{Tissue specimens}

Tissue samples were collected from 151 patients with ovarian serous adenocarcinoma during their initial treatment at the Chinese Academy of Medical Sciences Cancer Hospital (Beijing, China). All cancer tissues were diagnosed as primary ovarian cancer by histopathology. Patients received platinum-based adjuvant chemotherapy treatment after the first cytoreductive surgery and signed informed consent forms for sample collection to participate in this research. This study was approved by the Institutional Review Board of the Chinese Academy of Medical Sciences Cancer Institute.

\section{Immunohistochemistry}

An ovarian cancer tissue microarray including primary ovarian tumors was constructed with each case represented twice. For immunohistochemistry (IHC) staining, the slides were deparaffinized, rehydrated, immersed in 3\% hydrogen peroxide solution for 10 minutes, heated in a microwave in citrate buffer ( $\mathrm{pH}$ 6.0) for 10 minutes, and cooled at room temperature. The slides were blocked by $10 \%$ normal goat serum at $37^{\circ} \mathrm{C}$ for 30 minutes and then incubated with rabbit polyclonal antibody against XIAP at a dilution of 1:500 overnight at $4^{\circ} \mathrm{C}$. IHC was performed using the PV-9000 Polymer Detection System for Immuno-Histological Staining kit (Beijing Golden Bridge Biotechnology Company). 3,3'-Diaminobenzidine was used to visualize the reaction, followed by counterstaining with hematoxylin. Visual analysis was performed using ImageScope software (Aperio Technologies). The percentage of staining was automatically assessed by ImageScope software, and the expression score was determined by multiplying the percentage of staining by the staining intensity. Based on the median IHC score, tumors with scores were classified into the high-expression group (marked 1) and the low-expression group (recorded as 0 ). The location and scoring of the tumor were completed with two professional clinicopathologists.

\section{Cell lines and cell culture}

Ovarian carcinoma cell lines (ES2, SKOV3, 3AO) and 293 T cells were preserved in our laboratory. The ovarian cancer cell lines were cultured with RPMI-1640 medium containing 10\% FBS, $100 \mathrm{U} / \mathrm{mL}$ penicillin, and $100 \mathrm{mg} / \mathrm{mL}$ streptomycin. $293 \mathrm{~T}$ cells were maintained with DMEM supplemented with $10 \% \mathrm{FBS}, 100 \mathrm{U} / \mathrm{mL}$ penicillin, and $100 \mathrm{mg} / \mathrm{mL}$ streptomycin. All cells were cultured at $37^{\circ} \mathrm{C}$ in a humidified atmosphere containing $5 \% \mathrm{CO}_{2}$.

\section{Antibodies}

The immunohistochemistry antibody for XIAP (PRS3331) and the specific antibody for $\beta$-actin (A5316) were purchased from Sigma-Aldrich Co (St Louis, MO, USA). The antibody against caspase $3(9662 \mathrm{~S})$ and caspase 9 (9502S) were from Cell Signaling Technology. Specific antibodies against PARP (Sc-7150) and XIAP (Sc-55551) were purchased from Santa Cruz Biotechnology Inc. (Dallas, TX, USA).

\section{Packaging of virus and transfection}

The shGFP or shXIAP lentiviral and packaging vectors were transfected into $293 \mathrm{~T}$ cells according to manufacturer's instructions. The shRNA sequences were as follows: shXIAP-4: ACACGTACTTGTGCGAATTAT; shXIAP-6: GACTCTACTACACAGGTATTG; shGFP: GAGAAGCG CGATCACAT. Viral supernatants were collected after 48 hours and filtered through a $0.45 \mu \mathrm{m}$ filter. ES2 or $3 \mathrm{AO}$ cells were seeded in six-well plate $\left(2 \times 10^{5} /\right.$ well $)$ and infected with shGFP, shXIAP-4, and shXIAP-6 lentiviral particles, and then the cells were selected with puromycin $(2 \mu \mathrm{g} / \mathrm{mL})$. The knockdown efficiency of XIAP was confirmed by quantitative real-time PCR and Western blot.

\section{$I C_{50}$ value determination}

Ovarian cancer cells were seeded in 96-well plate $\left(1 \times 10^{4} /\right.$ well) and treated with carboplatin concentrations ranging from $40 \mu \mathrm{g} / \mathrm{mg}$ to $360 \mu \mathrm{g} / \mathrm{mg}$ for 24 hours. The cell viability was measured by Cell Counting Kit-8 (CCK-8) assay. Halfmaximal inhibitory concentration $\left(\mathrm{IC}_{50}\right)$ value of ovarian cancer cells was calculated by GraphPad Prism 6 software.

\section{Quantitative real-time PCR}

Total RNAs were extracted by TRIzol reagent (Thermo Fisher Scientific, Waltham, MA, USA). One microgram of total RNAs was reverse-transcribed to cDNA by using Quantscript RT Kit (Tiangn, Beijing, China). The expression of XIAP and GAPDH was analyzed by quantitative 
real-time PCR (RT-qPCR) with Power SYBR Green PCR Master Mix (Thermo Fisher Scientific). Primer sequences of XIAP and GAPDH were as follows: XIAP forward: 5'-GGACCCTCCCCTTGGAC-3' and reverse: 5'-TGAT GTCTGCAGGTACACAAGTT-3'; GAPDH forward: $5^{\prime}$-GGT GAAGGTCGGAGTCAACG- $3^{\prime}$ and reverse $5^{\prime}$-TGGGTGG AATCATATTGGAACA-3'.

\section{Western blotting}

Cells were lysed with lysis buffer on ice and centrifuged at $12,000 \mathrm{rpm}$ at $4^{\circ} \mathrm{C}$ for 20 minutes to collect the supernatant. Protein was quantified using bicinchoninic acid protein assay reagent. Protein samples were separated by SDS-PAGE and transferred to polyvinylidene difluoride membranes. Then, the membranes were blocked with 5\% milk for 1 hour and subsequently incubated with primary antibodies at $4{ }^{\circ} \mathrm{C}$ overnight, washed with TBST (Tris-buffered saline containing $0.05 \%$ Tween-20) for three times, followed by incubation with secondary antibodies. The membranes were washed with TBST for three times. Images were exposed by LAS4000 imaging system (Fujifilm, Valhalla, NY, USA).

\section{Cell proliferation assay}

Cells were seeded in 96 -well plate $\left(1 \times 10^{4} /\right.$ well $)$ and treated with vehicle control or carboplatin $(80 \mu \mathrm{g} / \mathrm{mL}$ or $200 \mu \mathrm{g} / \mathrm{mL})$. Cell proliferation was detected at 0,24 , and 48 hours using CCK-8 reagents (Wbsbio, Beijing, China) according to the manufacturer's instructions.

\section{Flow cytometry analysis of apoptosis}

Cells were seeded in a six-well plate $\left(2 \times 10^{5} /\right.$ well $)$ and treated with carboplatin $(200 \mu \mathrm{g} / \mathrm{mL}$ or $400 \mu \mathrm{g} / \mathrm{mL})$ for 24 hours. Then, cells were harvested for analysis of apoptosis using Annexin V-propidium iodide apoptosis detection kit (BD Biosciences, Franklin Lakes, NJ, USA) according to the instructions. The cell apoptotic rate was detected by BD LSR II flow cytometry (BD Biosciences).

\section{Statistical analysis}

SPSS 21.0 and GraphPad Prism 6 were used for statistical analysis. Pearson's chi-squared test was used to detect the relationship between XIAP level and clinical parameters. Kaplan-Meier survival analysis was used to calculate the relationship between various factors and patient's short progression-free survival (PFS). Data were presented as mean values with SD. $P$-value $<0.05$ was considered to be statistically significant.

\section{Results High expression of XIAP was associated with poor prognosis and chemotherapy resistance in ovarian cancer}

It has been reported that XIAP is associated with cisplatin sensitivity in ovarian cancer, ${ }^{9-11}$ we therefore investigated the relationship between XIAP expression and carboplatin sensitivity in ovarian cancer. Immunohistochemistry was performed on primary ovarian cancer specimens derived from 151 patients. Using the median expression value of XIAP as a cutoff point, the cohort was divided into XIAP high-expression group and XIAP low-expression group. Statistical analysis demonstrated that higher expression of XIAP was associated with lower probability of carboplatin sensitivity (Figure 1A), and that XIAP expression was also associated with primary CA125 levels (Table 1).

Further analysis showed that higher expression of XIAP was closely related to the patient's short PFS (Figure 1B and Table 2).
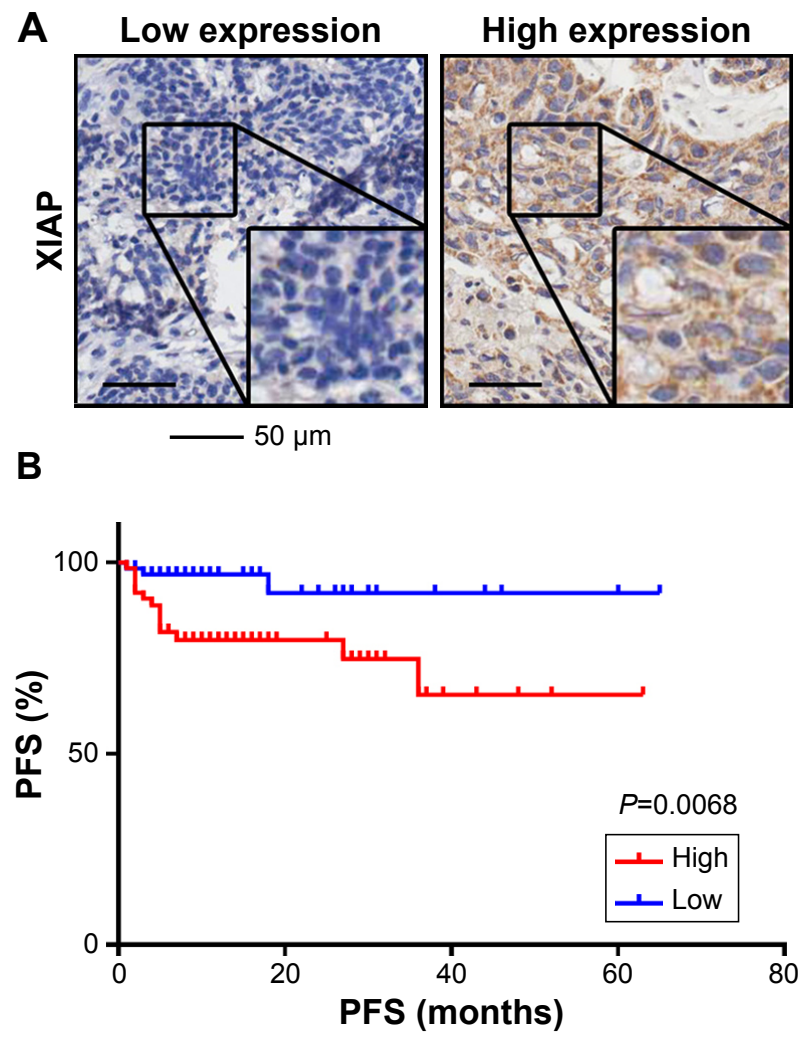

Figure I Relationship between XIAP expression and chemosensitivity in ovarian cancer Notes: (A) ScanScope Virtual Slide software was used to score the level of XIAP expression. Expression score at or below median was considered low and above median was considered high. (B) Kaplan-Meier plot shows that XIAP expression above median is associated with shorter PFS, $P=0.0068$.

Abbreviations: PFS, progression-free survival; XIAP, X-linked inhibitor of apoptosis protein. 
Table I Correlation between XIAP expression and clinical pathological features in SOC tissue microarray

\begin{tabular}{|c|c|c|c|c|}
\hline \multirow[t]{2}{*}{ Clinical variables } & \multirow[t]{2}{*}{$\begin{array}{l}\text { No of } \\
\text { patients }\end{array}$} & \multicolumn{2}{|c|}{$\begin{array}{l}\text { XIAP expression } \\
\text { (No of cases) }\end{array}$} & \multirow[t]{2}{*}{$P$-value } \\
\hline & & $\begin{array}{l}\text { Low } \\
(n=76)\end{array}$ & $\begin{array}{l}\text { High } \\
(n=75)\end{array}$ & \\
\hline All cases & 151 & & & \\
\hline \multicolumn{5}{|c|}{ Age at diagnosis (years) } \\
\hline$\geq 60$ & 45 & 21 & 24 & \multirow[t]{2}{*}{0.341} \\
\hline$<60$ & 106 & 55 & 51 & \\
\hline \multicolumn{5}{|c|}{ Residual tumor size $(\mathrm{cm})$} \\
\hline$\geq 2$ & 24 & 10 & 14 & \multirow[t]{2}{*}{0.241} \\
\hline$<2$ & 127 & 66 & 61 & \\
\hline \multicolumn{5}{|c|}{ Prime CAI 25 level $(\mathrm{U} / \mathrm{mL})$} \\
\hline$\geq 500$ & 115 & 63 & 52 & \multirow[t]{2}{*}{0.038} \\
\hline$<500$ & 36 & 13 & 23 & \\
\hline \multicolumn{5}{|l|}{ Recurrent type } \\
\hline Platinum-sensitive & 87 & 50 & 37 & \multirow[t]{2}{*}{0.03} \\
\hline Platinum-resistant & 64 & 26 & 38 & \\
\hline \multicolumn{5}{|l|}{2012 FIGO stage } \\
\hline I-II & 7 & 3 & 4 & \multirow[t]{2}{*}{0.493} \\
\hline III-IV & 144 & 73 & 71 & \\
\hline
\end{tabular}

Note: The relationship between XIAP expression level and clinical features was analyzed, $P<0.05$ was considered statistically significant.

Abbreviations: XIAP, X-linked inhibitor of apoptosis protein; SOC, serous ovarian cancer; CA, cancer antigen; FIGO, the International Federation of Gynecology and Obstetrics.

Table 2 Analyses of factors affecting PFS in patients with SOC microarray

\begin{tabular}{|c|c|c|}
\hline \multirow[t]{2}{*}{ Prognostic factors } & \multirow{2}{*}{$\begin{array}{l}\text { No of } \\
\text { cases }\end{array}$} & PFS \\
\hline & & $P$-value \\
\hline \multicolumn{3}{|c|}{ XIAP expression (IHC score) } \\
\hline Low $(0)$ & 76 & \multirow[t]{2}{*}{0.0068} \\
\hline High (I) & 75 & \\
\hline \multicolumn{3}{|l|}{ Age (years) } \\
\hline$\geq 60$ & 106 & \multirow[t]{2}{*}{0.1761} \\
\hline$<60$ & 24 & \\
\hline \multicolumn{3}{|l|}{ Tumor size $(\mathrm{cm})$} \\
\hline$\geq 2$ & 24 & \multirow[t]{2}{*}{$<0.0001$} \\
\hline$<2$ & 127 & \\
\hline \multicolumn{3}{|l|}{ CAI 25 level (U/mL) } \\
\hline$\geq 500$ & 115 & \multirow[t]{2}{*}{0.03275} \\
\hline$<500$ & 36 & \\
\hline \multicolumn{3}{|l|}{2012 FIGO stage } \\
\hline$I-I I$ & 7 & \multirow[t]{2}{*}{0.0065} \\
\hline III-IV & 144 & \\
\hline
\end{tabular}

Notes: The relationship between XIAP expression, clinical parameters, and progression-free survival (PFS) was analyzed. $P<0.05$ was considered statistically significant.

Abbreviations: PFS, progression-free survival; XIAP, X-linked inhibitor of apoptosis protein; IHC, immunohistochemistry; SOC, serous ovarian cancer; CA, cancer antigen; FIGO, the International Federation of Gynecology and Obstetrics.
Therefore, we concluded that XIAP expression in ovarian cancer is associated with sensitivity to carboplatin in patients, thus affecting the prognosis of patients.

\section{Establishment of stable XIAP knockdown cell lines}

To further validate the relationship between XIAP and carboplatin sensitivity at the cellular level, we first tested the expression levels of XIAP in three ovarian cancer cells, ES2, $3 \mathrm{AO}$, and SKOV3, at both protein and mRNA levels. The expression of XIAP was very high in these cells (Figure 2A). We then generated ES2 and $3 \mathrm{AO}$ cell lines with stable XIAP
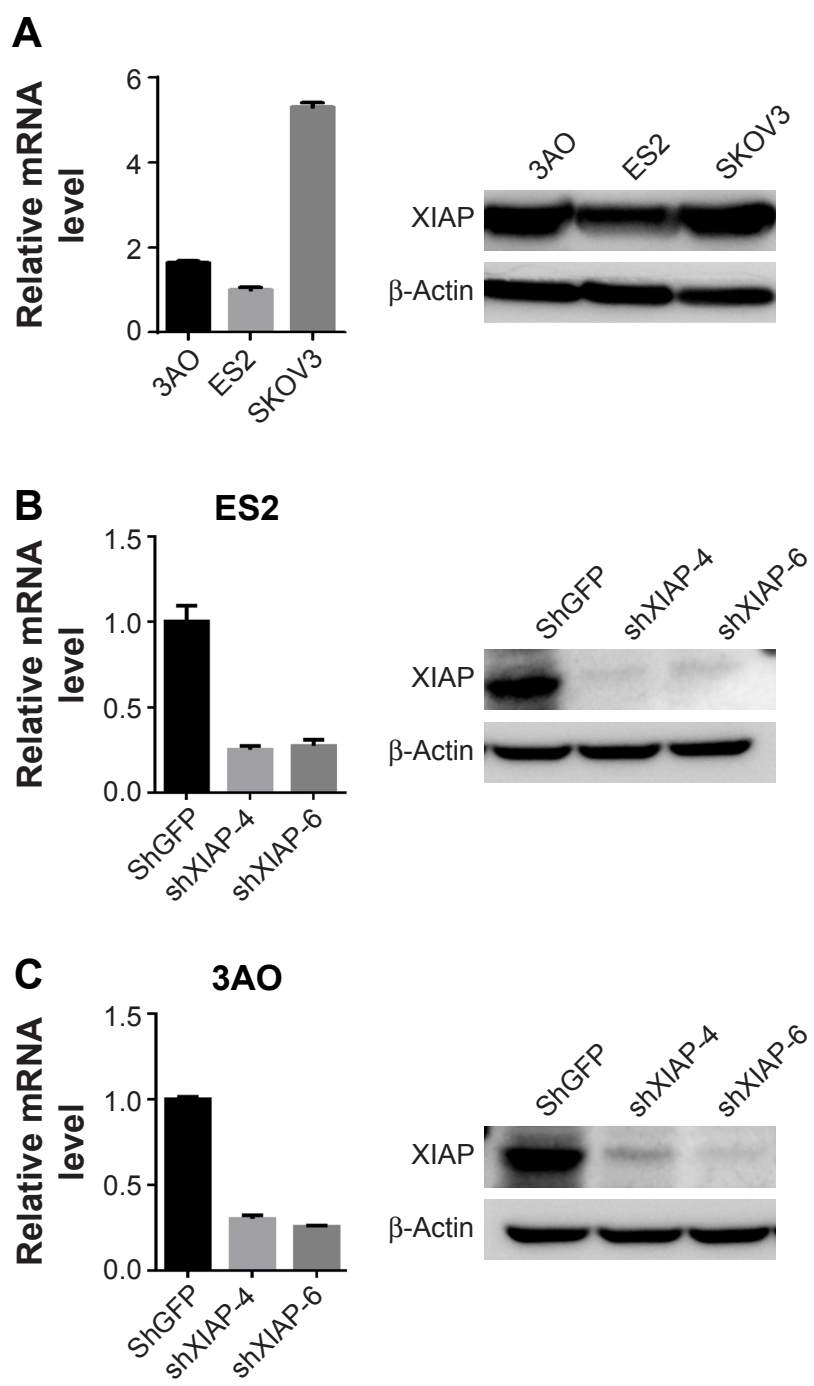

Figure 2 Establishment of stable knockdown of XIAP in ES2 and 3AO cells. Notes: (A) XIAP expression was examined by RT-qPCR (left panel) and Western blot (right panel) in various ovarian cancer cell lines. (B, C) ES2 (B) and 3AO (C) cells were infected with shGFP and shXIAP-4 and six lentivirus, and then stable cells were selected by puromycin $(2 \mu \mathrm{g} / \mathrm{mL})$. Cells were harvested, and XIAP expression was detected by RT-qPCR and Western blot.

Abbreviations: RT-qPCR, quantitative real-time PCR; XIAP, X-linked inhibitor of apoptosis protein. 
silencing by lentivirus-mediated RNA interference using two independent shRNA sequences (shXIAP-4 and shXIAP-6), and shGFP was used as control. The knockdown efficiency of XIAP was detected by RT-qPCR and Western blot. The results showed that XIAP expression was effectively suppressed (Figure 2B and C).

\section{Knockdown of XIAP retards cell proliferation}

To evaluate the cytotoxicity of carboplatin, we first evaluated the viabilities of ES2 and $3 \mathrm{AO}$ cells in a gradient carboplatin and determined the $\mathrm{IC}_{50}$ values of carboplatin in these cells (Figure 3A). Next, we investigated the inhibitory effect of carboplatin and assessed the role of XIAP in regulating carboplatin sensitivity. Based on the $\mathrm{IC}_{50}$ value, we treated stable XIAP knockdown ES2 and 3AO cells and their corresponding control cells with two concentrations of carboplatin ( $80 \mu \mathrm{g} / \mathrm{mL}$ or $200 \mu \mathrm{g} / \mathrm{mL}$ ), and then cell proliferation was detected by CCK-8 reagents. As expected, suppression of XIAP not only significantly retarded ES2 and $3 \mathrm{AO}$ proliferation but also sensitized cells to carboplatin-induced cell proliferation inhibition (Figure 3B and C).

\section{Knockdown of XIAP promotes carboplatin-induced cell apoptosis}

To investigate the effect of XIAP on carboplatin-induced apoptosis in ovarian cancer cells, we treated ES2 and $3 \mathrm{AO}$ cells with stable knockdown of XIAP and their corresponding control cells with different concentrations of carboplatin $(200 \mu \mathrm{g} / \mathrm{mL}$ or $400 \mu \mathrm{g} / \mathrm{mL})$. Flow cytometry
A

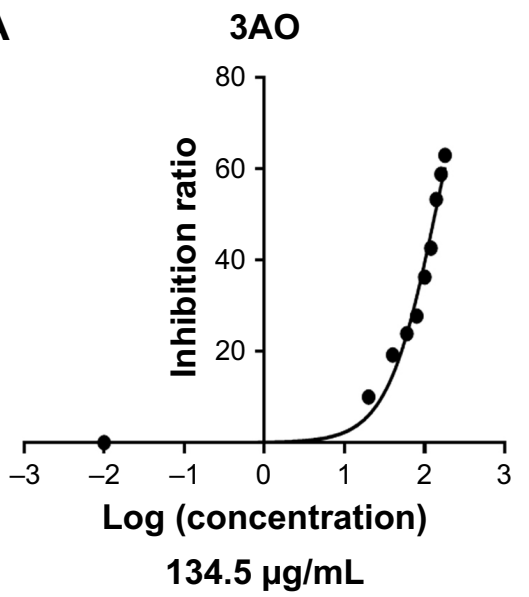

B

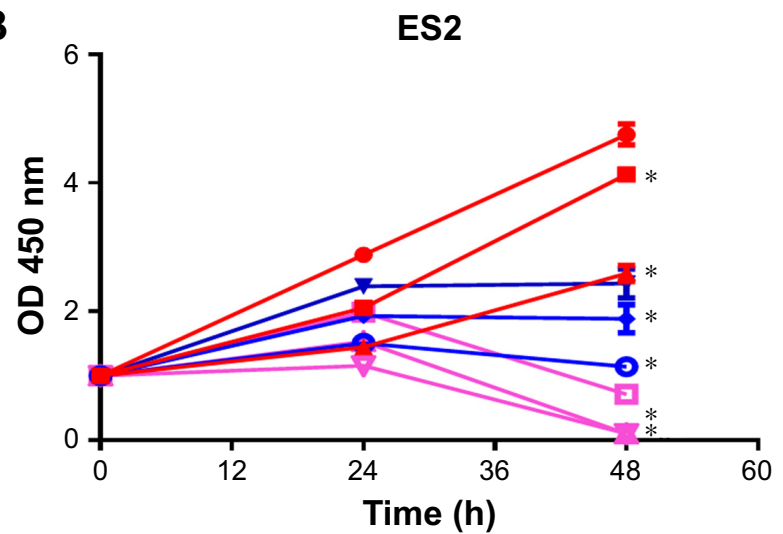

ES2

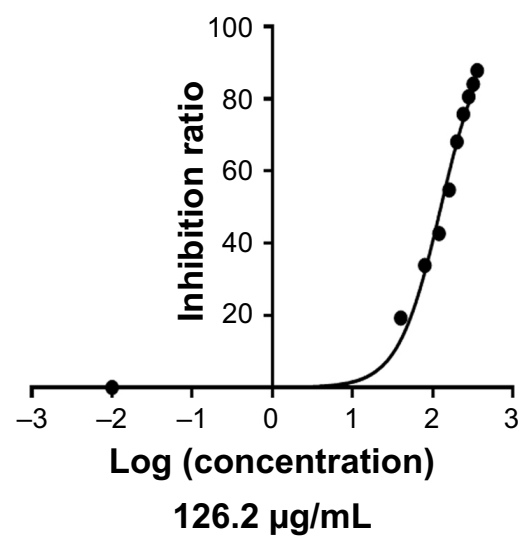

C

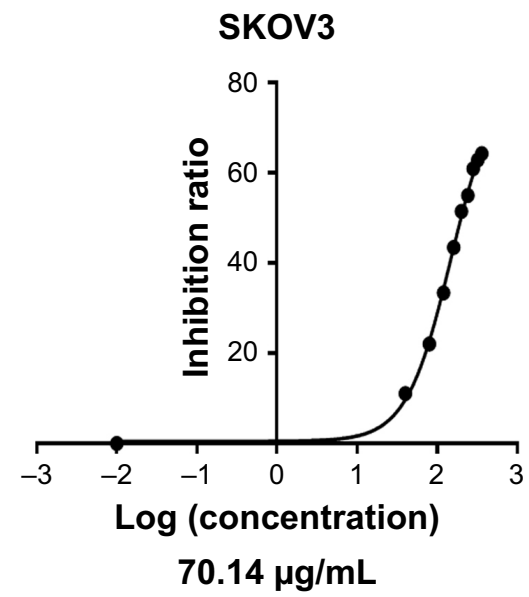

$70.14 \mu \mathrm{g} / \mathrm{mL}$

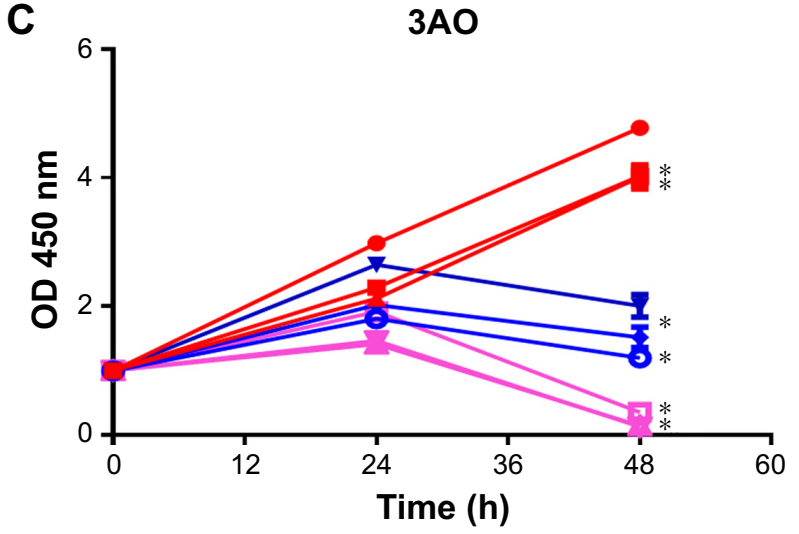

\begin{tabular}{|c|c|c|}
\hline - shGFP (vehicle) & - - shXIAP-4 (vehicle) & - shXIAP-6 (vehicle) \\
\hline$\neg$ shGFP (carbo-80 $\mu \mathrm{g} / \mathrm{mL}$ ) & $\multimap$ shXIAP-4 (carbo-80 $\mu \mathrm{g} / \mathrm{mL})$ & - - shXIAP-6 (carbo-80 $\mu \mathrm{g} / \mathrm{mL})$ \\
\hline -E- shGFP (carbo-200 $\mu \mathrm{g} / \mathrm{mL}$ ) & $\approx$ shXIAP-4 (carbo-200 $\mu \mathrm{g} / \mathrm{mL}$ ) & $\because$ shXIAP-6 (carbo-200 $\mu \mathrm{g} / \mathrm{mL})$ \\
\hline
\end{tabular}

Figure 3 Silencing of XIAP expression inhibits cell proliferation.

Notes: $(\mathbf{A}): \mathrm{IC}_{50}$ value of carboplatin was detected in three ovarian cancer cells. The IC $\mathrm{C}_{50}$ values of $3 \mathrm{AO}$, ES2, and SKOV3 were 134.5 , I26.2, and 70.14 $\mu \mathrm{g} / \mathrm{mL}$, respectively. (B, C) Cells were treated with carboplatin for 0,24 , and 48 hours, and CCK-8 assay was used to detect cell viability (B, ES2 cells; C, $3 A O$ cells). $* P<0.05$, $* * P<0.01$.

Abbreviations: $\mathrm{IC}_{50}$, half-maximal inhibitory concentration; XIAP, X-linked inhibitor of apoptosis protein. 
was used to determine cell apoptosis. Silencing of XIAP alone can significantly induce cell apoptosis in both cells. Moreover, suppression of XIAP further increased carboplatin-induced cell apoptosis (Figure 4A and B). Subsequently, the expression of apoptosis-related proteins (PARP, caspase 3, and caspase 9) was measured by
A
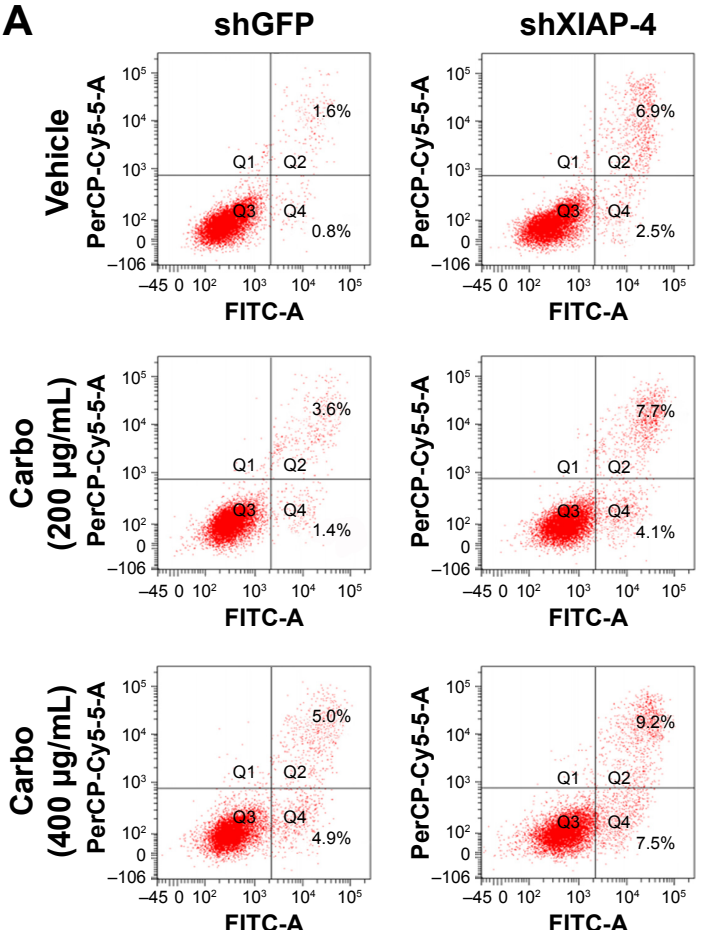

FITC-A

B
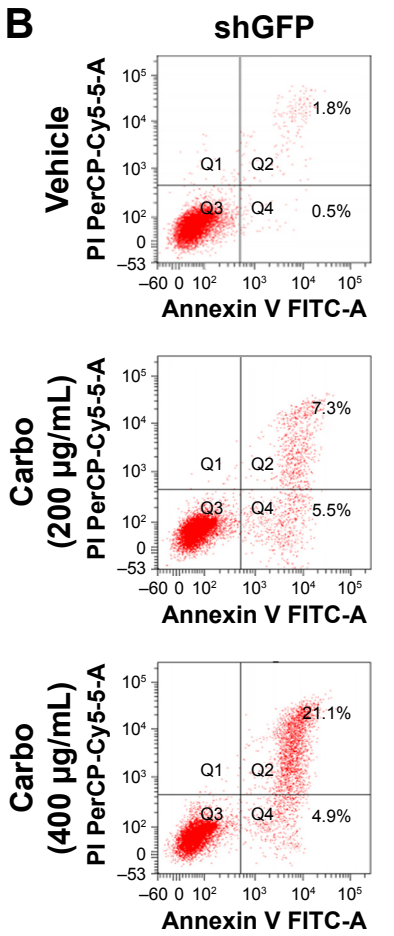

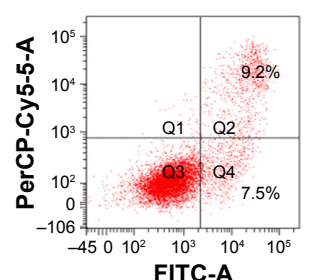

Annexin V
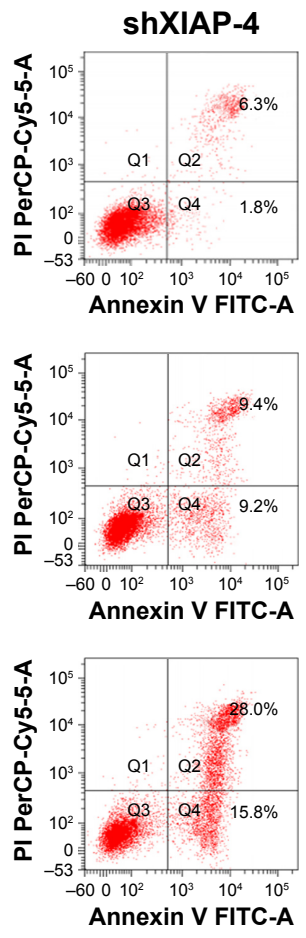
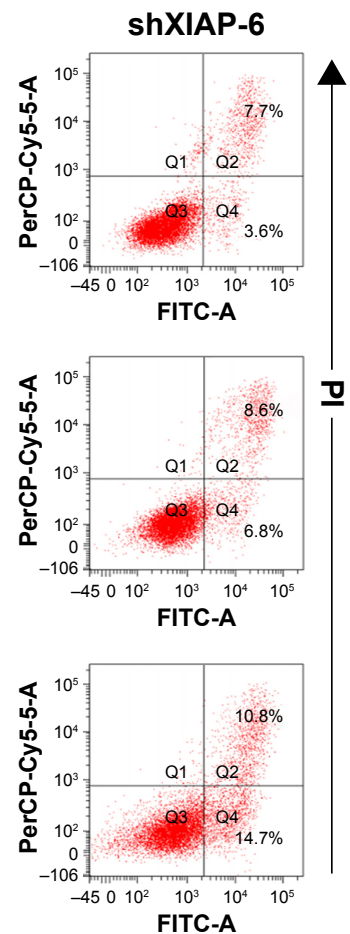

FITC-A
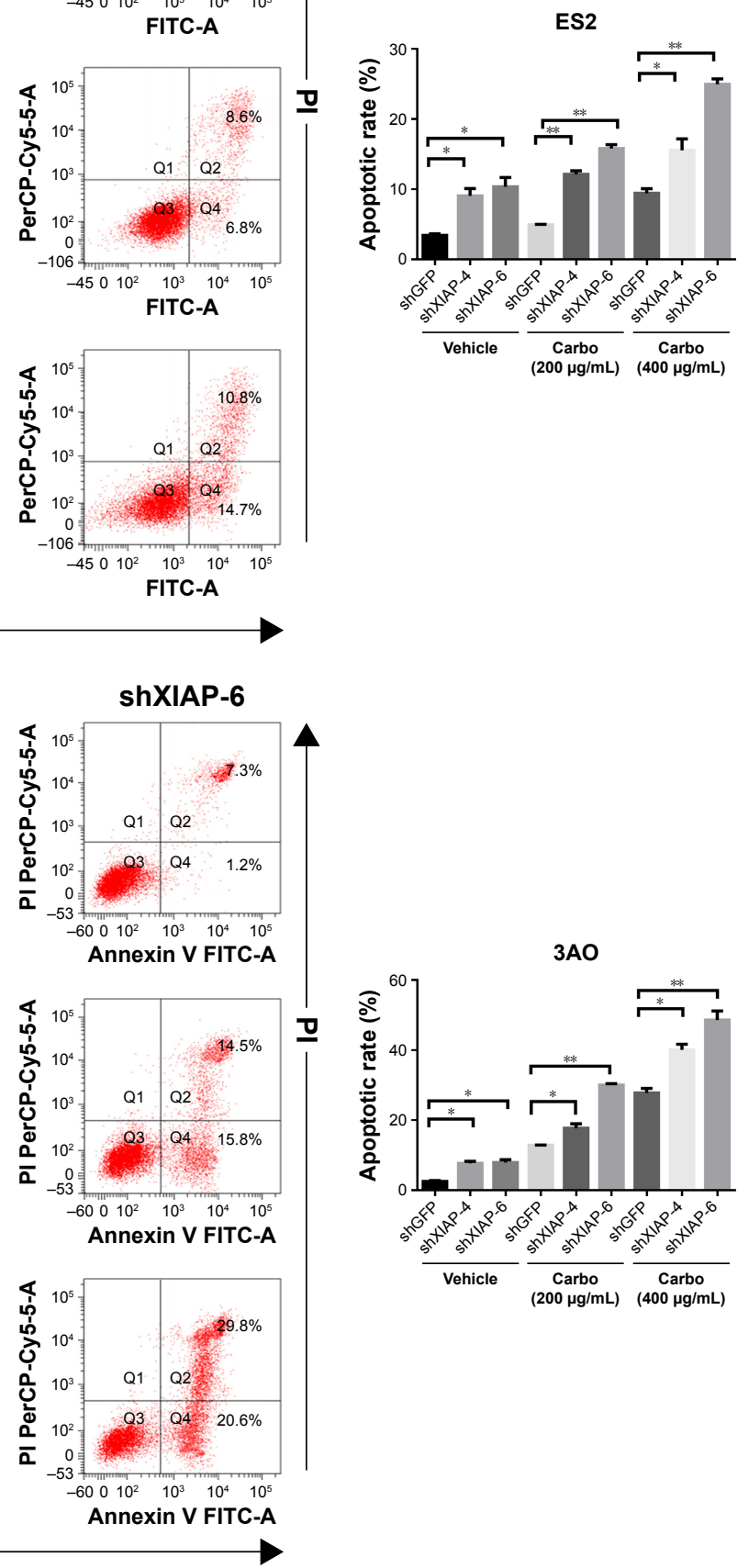

Figure 4 Knockdown of XIAP promotes cell apoptosis and sensitizes to carboplatin.

Notes: (A, B) Cells were treated with carboplatin for 24 hours, and apoptosis was detected using Annexin V-PI detection kit (A, ES2 cells; B, 3 AO cells). $* P<0.05$, $* * P<0.01$.

Abbreviations: PI, propidium iodide; XIAP, X-linked inhibitor of apoptosis protein. 


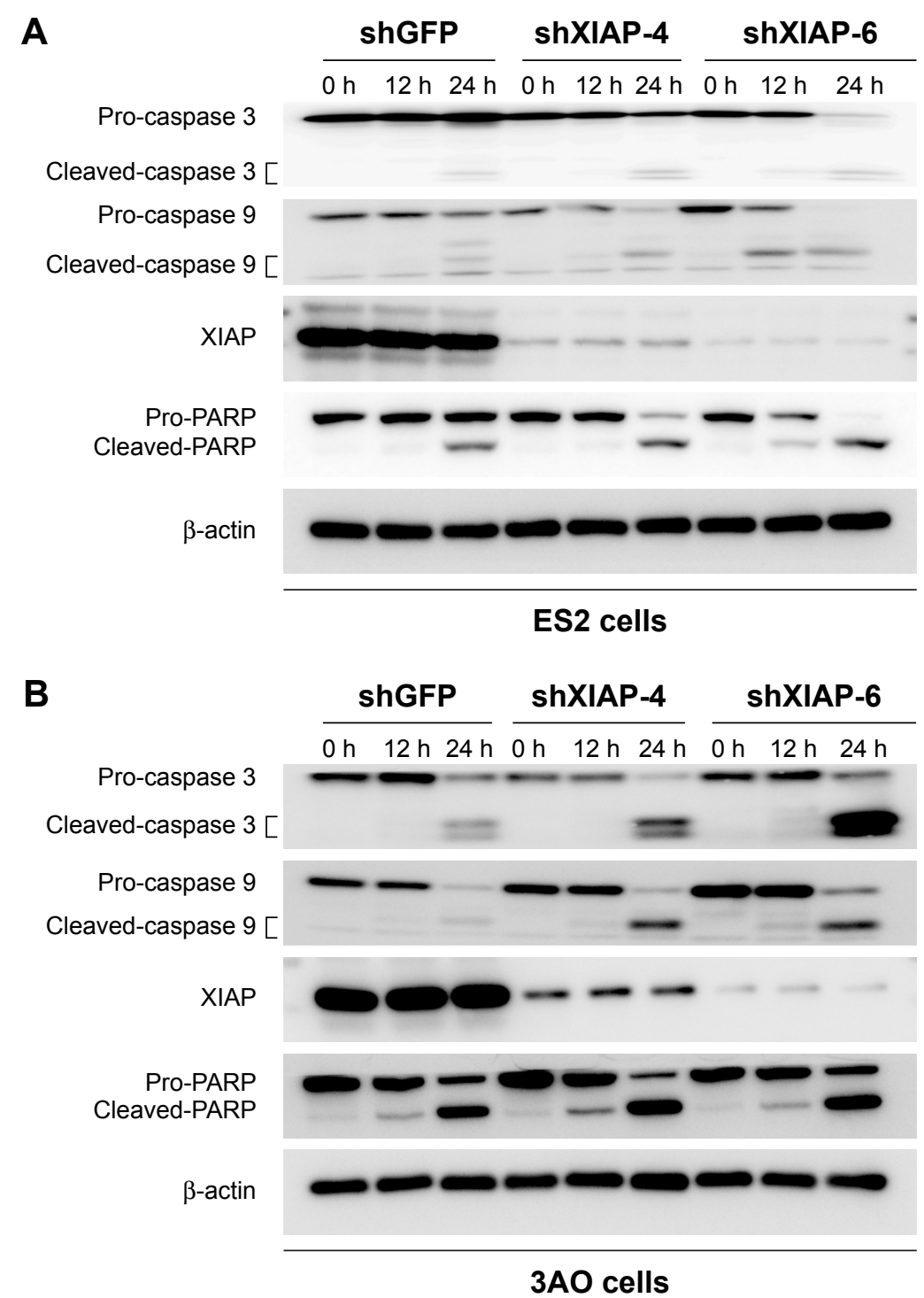

Figure 5 (A, B) Cells were treated with carboplatin at a concentration of $400 \mu \mathrm{g} / \mathrm{mL}$ for 0,12 , and 24 hours, and then the expression of apoptosis-related markers was detected by Western blot (A, ES2 cells; $\mathbf{B}, 3 \mathrm{AO}$ cells).

Abbreviation: XIAP, X-linked inhibitor of apoptosis protein.

Western blot. The results showed that attenuation of XIAP promoted carboplatin-induced cell apoptosis by increasing the cleavage of caspase 3, caspase 9, and PARP in ES2 and $3 \mathrm{AO}$ cells (Figure $5 \mathrm{~A}$ and $\mathrm{B}$ ). Taken together, these results suggest that XIAP is an important regulator of carboplatin sensitivity in ovarian cancer.

\section{Discussion}

Carboplatin is most commonly used as the front-line chemotherapeutic agent in ovarian carcinoma. However, carboplatin resistance is the major clinical obstacle for the treatment of ovarian cancer and the underlying mechanisms remain poorly understood. Therefore, investigating the mechanism of carboplatin resistance is undoubtedly important and is the basis for exploring novel strategies. In the current study, we first revealed that the expression of XIAP in ovarian cancer tissues was associated with carboplatin resistance and short PFS. We further provide strong evidence that XIAP suppression increased carboplatin chemosensitivity in ovarian cancer cells.

With recent progress in drug resistance, it has become clear that loss of control on cell death and apoptosis may confer chemoresistance. ${ }^{15,16}$ XIAP, a member of the IAP family, prevents apoptosis induced by both extrinsic and intrinsic pathways through direct inhibition of effector caspases (caspases 3, 6, 7, and 9). ${ }^{17}$ It has been well-documented that XIAP plays a crucial role in tumor maintenance and resistance to chemotherapy treatments in many kinds of tumors. ${ }^{13,18-20}$ Cells that lack XIAP are sensitized to apoptosis. High expression of XIAP has also been found in ovarian 
cancer cells and implicated as determinant of the chemosensitivity of ovarian cancer. ${ }^{21-24}$ Increasing evidence has focused on XIAP suppression to develop new strategies for cancer prevention and treatment. ${ }^{25}$ Consistent with previous studies, we demonstrated the role of XIAP in the protection of ovarian cancer cells induced by carboplatin. We also confirmed that XIAP inhibited carboplatin-induced apoptosis via suppression of caspases 3 and 9. In future studies, in vivo experimental evidence needs to be further provided to confirm the functional role of XIAP in mediating carboplatin sensitivity. Mechanistic investigations have demonstrated that a number of signaling pathways are involved in the regulation of XIAP expression, including p53, NF-KB, c-Jun/AP-1, PI3K, and USP11-dependent signaling pathways. ${ }^{5,20,26-28}$ The precise molecular biological mechanism as to how XIAP regulates apoptosis of ovarian cancer cell must also be addressed in the future.

\section{Conclusion}

Our data clearly demonstrate that XIAP suppression enhances chemosensitivity of carboplatin and that XIAP can act as a predictor of carboplatin sensitivity. Thus, XIAP-targeted regulation may markedly contribute to the identification of biomarkers of chemotherapy and present a promising therapeutic strategy to improve chemotherapeutic efficacy in ovarian cancer.

\section{Acknowledgment}

This work was supported by National Natural Science Foundation of China (81472452), Chinese Academy of Medical Sciences Initiative for Innovative Medicine (2017-I2M3-004), and Beijing Science and Technology Commission Project (K2015311201501).

\section{Author contributions}

Yiping Zhang, Zhihua Liu, Hongyan Chen and Yinghui Huang conceived the study, interpreted the results and wrote the manuscript. Yiping Zhang performed immunohistochemistry and analysis. Yiping Zhang, Furong Huang, Qingyu Luo and Xiaowei Wu performed laboratory experiments and statistical analyses. All authors contributed to data analysis, drafting and revising the article, gave final approval of the version to be published, and agree to be accountable for all aspects of the work.

\section{Disclosure}

The authors report no conflicts of interest in this work.

\section{References}

1. Tattersall MHN. Ovarian cancer chemotherapy: carboplatin as standard. Lancet. 2002;360(9332):500-501.

2. Agarwal R, Kaye SB. Ovarian cancer: strategies for overcoming resistance to chemotherapy. Nat Rev Cancer. 2003;3(7):502-516.

3. Stone RL, Sood AK, Coleman RL. Collateral damage: toxic effects of targeted antiangiogenic therapies in ovarian cancer. Lancet Oncol. 2010; 11(5):465-475.

4. Bookman MA, Gilks CB, Kohn EC, et al. Better therapeutic trials in ovarian cancer. J Natl Cancer Inst. 2014;106(4):dju029.

5. Gyrd-Hansen M, Darding M, Miasari M, et al. IAPs contain an evolutionarily conserved ubiquitin-binding domain that regulates NF-kappaB as well as cell survival and oncogenesis. Nat Cell Biol. 2008;10(11):1309-1317.

6. Mehrotra S, Languino LR, Raskett CM, Mercurio AM, Dohi T, Altieri DC. IAP regulation of metastasis. Cancer Cell. 2010;17(1):53-64.

7. Huang $X, W u Z$, Mei $Y, W u ~ M$. XIAP inhibits autophagy via XIAPMdm2-p53 signalling. Embo J. 2013;32(16):2204-2216.

8. Krieg A, Correa RG, Garrison JB, et al. XIAP mediates NOD signaling via interaction with RIP2. Proc Natl Acad Sci U S A. 2009;106(34): 14524-14529.

9. He X, Khurana A, Maguire JL, Chien J, Shridhar V. HtrA1 sensitizes ovarian cancer cells to cisplatin-induced cytotoxicity by targeting XIAP for degradation. Int J Cancer. 2012;130(5):1029-1035.

10. Farrand L, Byun S, Kim JY, et al. Piceatannol enhances cisplatin sensitivity in ovarian cancer via modulation of p53, X-linked inhibitor of apoptosis protein (XIAP), and mitochondrial fission. J Biol Chem. 2013; 288(33):23740-23750.

11. Miyamoto M, Takano M, Aoyama T, et al. Phenoxodiol Increases Cisplatin Sensitivity in Ovarian Clear Cancer Cells Through XIAP Down-regulation and Autophagy Inhibition. Anticancer Res. 2018;38(1):301-306.

12. Zhang X, Huang L, Zhao Y, Tan W. Downregulation of miR-130a contributes to cisplatin resistance in ovarian cancer cells by targeting X-linked inhibitor of apoptosis (XIAP) directly. Acta Biochim Biophys Sin (Shangai). 2013;45(12):995-1001.

13. Lin YF, Lai TC, Chang CK, et al. Targeting the XIAP/caspase-7 complex selectively kills caspase-3-deficient malignancies. J Clin Invest. 2013;123(9):3861-3875.

14. Zhao WJ, Deng BY, Wang XM, Miao Y, Wang JN. XIAP associated factor 1 (XAF1) represses expression of X-linked inhibitor of apoptosis protein (XIAP) and regulates invasion, cell cycle, apoptosis, and cisplatin sensitivity of ovarian carcinoma cells. Asian Pac J Cancer Prev. 2015;16(6):2453-2458.

15. Fernandez-Capetillo O, Chen HT, Celeste A, et al. DNA damageinduced G2-M checkpoint activation by histone H2AX and 53BP1. Nat Cell Biol. 2002;4(12):993-997.

16. Arts HJ, Van Der Zee AG, De Jong S, De Vries EG. Options for modulation of drug resistance in ovarian cancer. Int J Gynecol Cancer. 2000; 10(S1):47-52.

17. Gyrd-Hansen M, Meier P. IAPs: from caspase inhibitors to modulators of NF-kappaB, inflammation and cancer. Nat Rev Cancer. 2010; 10(8):561-574

18. Gu L, Zhu N, Zhang H, Durden DL, Feng Y, Zhou M. Regulation of XIAP translation and induction by MDM2 following irradiation. Cancer Cell. 2009;15(5):363-375.

19. Tosello V, Bordin F, Yu J, et al. Calcineurin and GSK-3 inhibition sensitizes T-cell acute lymphoblastic leukemia cells to apoptosis through X-linked inhibitor of apoptosis protein degradation. Leukemia. 2016; 30(4):812-822.

20. Schile AJ, García-Fernández M, Steller H. Regulation of apoptosis by XIAP ubiquitin-ligase activity. Genes Dev. 2008;22(16):2256-2266.

21. Fraser M, Leung BM, Yan X, Dan HC, Cheng JQ, Tsang BK. p53 is a determinant of X-linked inhibitor of apoptosis protein/Akt-mediated chemoresistance in human ovarian cancer cells. Cancer Res. 2003; 63(21):7081-7088. 
22. Asselin E, Mills GB, Tsang BK. XIAP regulates Akt activity and caspase-3-dependent cleavage during cisplatin-induced apoptosis in human ovarian epithelial cancer cells. Cancer Res. 2001;61(5):1862-1868.

23. Li J, Feng Q, Kim JM, et al. Human ovarian cancer and cisplatin resistance: possible role of inhibitor of apoptosis proteins. Endocrinology. 2001;142(1):370-380.

24. Sasaki H, Sheng Y, Kotsuji F, Tsang BK. Down-regulation of X-linked inhibitor of apoptosis protein induces apoptosis in chemoresistant human ovarian cancer cells. Cancer Res. 2000;60(20):5659-5666.

25. Chaudhary AK, Yadav N, Bhat TA, O’Malley J, Kumar S, Chandra D. A potential role of $\mathrm{X}$-linked inhibitor of apoptosis protein in mitochondrial membrane permeabilization and its implication in cancer therapy. Drug Discov Today. 2016;21(1):38-47.
26. Zhou Z, Luo A, Shrivastava I, et al. Regulation of XIAP Turnover Reveals a Role for USP11 in Promotion of Tumorigenesis. EBioMedicine. 2017;15:48-61.

27. Cao Z, Zhang R, Li J, et al. X-linked inhibitor of apoptosis protein (XIAP) regulation of cyclin D1 protein expression and cancer cell anchorage-independent growth via its E3 ligase-mediated protein phosphatase 2A/c-Jun axis. J Biol Chem. 2013;288(28):20238-20247.

28. Rada M, Nallanthighal S, Cha J, et al. Inhibitor of apoptosis proteins (IAPs) mediate collagen type XI alpha 1-driven cisplatin resistance in ovarian cancer. Oncogene. 2018;37(35):4809-4820.

\section{Publish your work in this journal}

OncoTargets and Therapy is an international, peer-reviewed, open access journal focusing on the pathological basis of all cancers, potential targets for therapy and treatment protocols employed to improve the management of cancer patients. The journal also focuses on the impact of management programs and new therapeutic agents and protocols on

\section{Dovepress}

patient perspectives such as quality of life, adherence and satisfaction. The manuscript management system is completely online and includes a very quick and fair peer-review system, which is all easy to use. Visit http://www.dovepress.com/testimonials.php to read real quotes from published authors.

Submit your manuscript here: http://www.dovepress.com/oncotargets-and-therapy-journal 